\title{
A Polynomial Solution to the Model Matching Problem of Nonlinear Time-delay Systems
}

\author{
Miroslav Halás and Claude H. Moog
}

\begin{abstract}
In this paper the model matching problem is considered for single input single output nonlinear systems with delays. A full characterization for its solvability is provided within a simple class of compensators. The approach is developed by means of the transfer functions of nonlinear time-delay systems. The state elimination problem for the systems given by their state-space representation is discussed as well.
\end{abstract}

\section{INTRODUCTION}

An algebraic formalism of differential forms, originally developed for nonlinear systems without delays [6], [2], was recently extended to the case of time-delay systems [22], [24], [25], [26], [31] and was shown to be effective in solving control problems like accessibility and observability, disturbance decoupling, feedback linearization and others.

On the other side, in the case of systems without delays, there exists, in comparison to the machinery of one-forms, an alternative approach in which the system properties are described by skew polynomials from non-commutative polynomial rings. Such polynomials act as differential [33], [34] or shift [20] operators on the differentials of the system inputs and outputs. The polynomial approach to nonlinear systems shows a great similarity to methods well known from the linear theory, see for instance [30]. In particular, one can introduce even a notion of a transfer function of a nonlinear system as was recently shown in [10], [13], [14], [15], [32]. Such a concept is equivalent to that of [7] for linear timevarying systems which allows us to associate to a nonlinear system the tangent (or variational) linear system, see for instance [8], over Kähler differentials [19] except that now the time-varying coefficients of the polynomials are not necessarily independent [21]. The transfer function formalism of nonlinear systems generalizes well known results valid for linear time invariant systems and was, for instance, already employed in [29] to investigate some structural properties of nonlinear systems, in [18] to study the nonlinear model matching problem, in [16] to study the observer design and in [17] to study the realization problem of nonlinear systems. The polynomial approach to nonlinear systems and the notion of a transfer function was recently carried over also to the case of time-delay systems [11], [12] where some basic

M. Halás is with Institute of Control and Industrial Informatics, Fac. of Electrical Engineering and IT, Slovak University of Technology, Ilkovičova 3, 81219 Bratislava, Slovakia. miroslav.halas estuba.sk Partially supported by the ESF project JPD 3 2005/NP1-047, code No. 13120200115, and the Slovak Research and Development Agency, contract No. LPP-0127-06.

C. H. Moog is with IRCCyN, UMR C.N.R.S. 6597, 1 rue de la Noë, BP 92101, 44321 Nantes Cedex 3, France. moogeirccyn.ec-nantes.fr properties were discussed. In this paper, the model matching problem of nonlinear time-delay systems is solved within such a transfer function formalism. As it was done in [12], a more detailed discussion is provided below, concerning also structural properties as the notions of a relative degree and a relative shift. In addition, we also depict a possible solution to the state elimination problem for the nonlinear time-delay case.

\section{TRANSFER FUNCTIONS OF NONLINEAR TIME-DELAY SYSTEMS}

We will use the algebraic formalism of [22], [24], [25], [26], [31] which extends the concept of differential oneforms to the case of time-delay systems and of [11], [12] which introduces transfer functions of nonlinear time-delay systems.

In this paper we restrict our attention to the SISO nonlinear time-delay systems defined by an input-output equation of the form

$$
y^{(n)}(t)=\varphi\left(\left\{y^{(k)}(t-i), u^{(l)}(t-j)\right\}\right)
$$

where $0 \leq k \leq n-1 ; 0 \leq l \leq n ; i, j \geq 0$ and $u \in \mathbf{R}$ and $y \in \mathbf{R}$ denote input and output to the system and $\varphi$ is assumed to be an element of the field of meromorphic functions $\mathcal{K}$.

Note that it is not restrictive to assume $i, j \in \mathbf{N}$ as all commensurable delays can be considered as multiples of an elementary delay $h$ [25].

Remark 1: In the case of systems without delays even if one starts with a state-space representation it is always possible to eliminate the state variables to get an input-output equation, see for instance [6]. However, it is not completely clear how one can carry over the idea of the state elimination procedure to the time-delay case. A special case is discussed in Appendix I. In the general case, possible drawbacks may be met in the state elimination process for system (10). For the sake of simplicity, here we assume that the system (10) admits an input-output equation of the form (1).

The Ore algebra $\mathcal{K}[\delta, s]$ of polynomials in $s$ and $\delta$ over $\mathcal{K}$ with the usual addition, and the (non-commutative) multiplications given by the commutation rules

$$
\begin{aligned}
s a(t) & =a(t) s+\dot{a}(t) \\
\delta a(t) & =a(t-1) \delta \\
s \delta & =\delta s
\end{aligned}
$$

where $a(t) \in \mathcal{K}$, represents the ring of linear differential time-delay operators that act over the vector space of one- 
forms $\mathcal{E}=\operatorname{span}_{\mathcal{K}}\{\mathrm{d} \xi(t) ; \xi(t) \in \mathcal{K}\}$ in the following way

$$
\left(\sum_{i, j} a_{i j} \delta^{j} s^{i}\right) v(t)=\sum_{i, j} a_{i j} v^{(i)}(t-j)
$$

for any $v(t) \in \mathcal{E}$.

The commutation rules (2) actually represent the rule for differentiating and, respectively, time-delaying.

Proposition 1 (Ore condition): For all non-zero $a, b \in$ $\mathcal{K}[\delta, s]$, there exist non-zero $a_{1}, b_{1} \in \mathcal{K}[\delta, s]$ such that $a_{1} b=$ $b_{1} a$.

Thus, the ring $\mathcal{K}[\delta, s]$ can be embedded to the noncommutative quotient field $\mathcal{K}\langle\delta, s\rangle$ by defining quotients [27], [28] as

$$
\frac{a}{b}=b^{-1} \cdot a
$$

The addition and multiplication in $\mathcal{K}\langle\delta, s\rangle$ are defined as

$$
\frac{a_{1}}{b_{1}}+\frac{a_{2}}{b_{2}}=\frac{\beta_{2} a_{1}+\beta_{1} a_{2}}{\beta_{2} b_{1}}
$$

where $\beta_{2} b_{1}=\beta_{1} b_{2}$ by Ore condition and

$$
\frac{a_{1}}{b_{1}} \cdot \frac{a_{2}}{b_{2}}=\frac{\alpha_{1} a_{2}}{\beta_{2} b_{1}}
$$

where $\beta_{2} a_{1}=\alpha_{1} b_{2}$ again by Ore condition.

Due to the non-commutative multiplications (2) they, of course, differ from the usual rules. In particular, in case of the multiplication (3) we, in general, cannot simply multiply numerators and denominators, nor cancel them in a usual manner. We neither can commute them as the multiplication in $\mathcal{K}\langle\delta, s\rangle$ is non-commutative as well.

Once the fraction of two skew polynomials is defined we can introduce the transfer function of the nonlinear timedelay system (1) as an element $F(\delta, s) \in \mathcal{K}\langle\delta, s\rangle$ such that $\mathrm{d} y(t)=F(\delta, s) \mathrm{d} u(t)$.

After differentiating (1) we get

$$
\begin{aligned}
\mathrm{d} y^{(n)}(t) & -\sum_{\substack{k=0 \\
i \geq 0}}^{n-1} \frac{\partial \phi}{\partial y^{(k)}(t-i)} \mathrm{d} y^{(k)}(t-i)= \\
& =\sum_{\substack{k=0 \\
j \geq 0}}^{n-1} \frac{\partial \phi}{\partial u^{(k)}(t-j)} \mathrm{d} u^{(k)}(t-j)
\end{aligned}
$$

or alternatively

$$
a(\delta, s) \mathrm{d} y(t)=b(\delta, s) \mathrm{d} u(t)
$$

where $a(\delta, s)=s^{n}-\sum_{k=0, i \geq 0}^{n-1} \frac{\partial \phi}{\partial y^{(k)}(t-i)} \delta^{i} s^{k}$ and $b(\delta, s)=$ $\sum_{k=0, j \geq 0}^{n-1} \frac{\partial \phi}{\partial u^{(k)}(t-j)} \delta^{j} s^{k}$ are in $\mathcal{K}[\delta, s]$. Then

$$
\begin{aligned}
F(\delta, s) & =\frac{b(\delta, s)}{a(\delta, s)} \\
& =\frac{b_{m}(\delta) s^{m}+\cdots+b_{1}(\delta) s+b_{0}(\delta)}{s^{n}+a_{n-1}(\delta) s^{n-1}+\cdots+a_{1}(\delta) s+a_{0}(\delta)}
\end{aligned}
$$

where $m=\max \left\{i \geq 0 ; \partial \varphi / \partial u^{(i)} \neq 0\right\}$.

Example 1: Consider the system

$$
\ddot{y}(t)=\dot{y}(t-1) u(t-1)
$$

After differentiating

$$
\begin{aligned}
\mathrm{d} \ddot{y}(t)-u(t-1) \mathrm{d} \dot{y}(t-1) & =\dot{y}(t-1) \mathrm{d} u(t-1) \\
\left(s^{2}-u(t-1) \delta s\right) \mathrm{d} y(t) & =\dot{y}(t-1) \delta \mathrm{d} u(t)
\end{aligned}
$$

and the transfer function is

$$
F(\delta, s)=\frac{\dot{y}(t-1) \delta}{s^{2}-u(t-1) \delta s}
$$

The reader is referred to [10], [11], [12], [13], [14], [15], [18] for more detailed description of the transfer function formalism in nonlinear control theory and to [3], [4], [5], [11], [12] for more detailed description of Ore rings and Ore algebras.

\section{STRUCTURAL PROPERTIES}

Transfer functions of control systems are well known from linear theory. Their use is the most natural for a several control problems, for they give the insight into the system structure. Recently, it was shown that such an algebraic formalism can be generalized also to nonlinear systems without delays [10], [14] and even to the time-delay case [12]. In this section we, in comparison to [12], discuss additional structural properties of transfer functions of nonlinear timedelay systems.

In dealing with polynomials from $\mathcal{K}[\delta, s]$ we define their (highest) degrees and lowest degrees in $s$ and in $\delta$ as

$$
\begin{aligned}
\operatorname{deg}_{s} a(\delta, s) & =\max \left\{k \geq 0 ; \frac{\partial a(\delta, s)}{\partial s^{k}} \neq 0\right\} \\
\operatorname{deg}_{\delta} a(\delta, s) & =\max \left\{k \geq 0 ; \frac{\partial a(\delta, s)}{\partial \delta^{k}} \neq 0\right\} \\
\text { low } \operatorname{deg}_{s} a(\delta, s) & =\min \left\{k \geq 0 ; \frac{\partial a(\delta, s)}{\partial s^{k}} \neq 0\right\} \\
\text { low } \operatorname{deg}_{\delta} a(\delta, s) & =\min \left\{k \geq 0 ; \frac{\partial a(\delta, s)}{\partial \delta^{k}} \neq 0\right\}
\end{aligned}
$$

where $0 \neq a(\delta, s) \in \mathcal{K}[\delta, s]$. If $a(\delta, s)=0$ then define $\operatorname{deg}_{s} a(\delta, s)=\operatorname{deg}_{\delta} a(\delta, s)=-\infty$ and $\operatorname{low}_{\operatorname{deg}_{s} a(\delta, s)=}$ low $\operatorname{deg}_{\delta} a(\delta, s)=\infty$.

\section{A. Structural indices}

Certain structural indices of a control system play a key role in solving a number of control problems. One of them is represented by the notion of a relative degree, originally introduced for the case of systems without delays. It can be easily extended also to the time-delay case [26]:

Definition 1: The relative degree of the system (10) is defined as

$$
\rho=\min \left\{k \geq 0 ; \frac{\partial y^{(k)}(t)}{\partial u(t-j)} \neq 0 \text { for some } j \geq 0\right\}
$$

It is straightforward to show that the relative degree is given as the difference between orders of polynomials in $s$ in denominator and numerator of the transfer function (5)

$$
\rho=\operatorname{rel} \operatorname{deg}_{s} F(\delta, s)=\operatorname{deg}_{s} a(\delta, s)-\operatorname{deg}_{s} b(\delta, s)
$$

as in the linear case.

Another important structural index in the time-delay case is given by the notion of a relative shift [24], [26]: 
Definition 2: Assume that the relative degree $\rho$ of the system (10) is finite. Then the relative shift of this system is defined as

$$
\mu=\min \left\{k \geq 0 ; \frac{\partial y^{(\rho)}(t)}{\partial u(t-k)} \neq 0\right\}
$$

It can be shown that in terms of the transfer function (5) it means

$$
\mu=\operatorname{deg}_{\delta} b_{m}(\delta)
$$

where $b_{m}(\delta)$ is the leading coefficient of $b(\delta, s)$ in $s$.

Example 2: Consider the system from Example 1 with the transfer function (6). Then

$$
\begin{aligned}
\rho & =\operatorname{reldeg}_{s} F(\delta, s)=2 \\
\mu & =\operatorname{deg}_{\delta} \dot{y}(t-1) \delta=1
\end{aligned}
$$

Remark 2: Note that in comparison to the relative degree, the relative shift is not the difference between orders of polynomials in $\delta$ in denominator and numerator of the transfer function (5), $\mu \neq \operatorname{rel}_{\operatorname{deg}_{\delta}} F(\delta, s)=\operatorname{deg}_{\delta} a(\delta, s)-$ $\operatorname{deg}_{\delta} b(\delta, s)$, which does not play such an important role as the notion of the relative shift.

\section{B. Accessibility}

An important notion, concerning additional structural properties of a given system, is the notion of accessibility. It is related mainly to the possibility of controlling the system and can be carried over also to the time-delay case [22], [23]:

Definition 3: The system (1) is said to be accessible if there does not exist any non-constant autonomous function.

One possibility how to study the accessibility is to introduce the notion of an autonomous element. In the case of system without delays this results in the equivalence of the two following conditions:

- There does not exist any non-constant autonomous function for system.

- System does not have any autonomous elements.

For linear time-delay systems both definitions are equivalent to the property that the system is torsion free over the ring $\mathbf{R}[\delta, \mathrm{d} / \mathrm{d} t],[9]$. However, in the case of nonlinear time-delay systems their equivalence remains an open problem [23].

The accessibility filtration, employing the notion of an autonomous element, was introduced for nonlinear systems without delays in [6]. Recently, it was extended to the timedelay case as well [22], [23]. However, it should be noticed that the submodules $\mathcal{H}_{k}$ are, in general, infinite dimensional for time-delay systems, which represents a major drawback of their practical computation. Therefore, an alternative way to compute this filtration was studied [23].

However, another alternative accessibility condition can be stated in terms of polynomials $a(\delta, s)$ and $b(\delta, s)$ in (4).

Proposition 1: The system (1) is accessible if polynomials $a(\delta, s)$ and $b(\delta, s)$ have no nontrivial common left factors.

Proof: It was shown in [11] that $a(\delta, s)$ and $b(\delta, s)$ have no common left factors if and only if system does not have any autonomous elements, following the same line as in [33].

\section{Observability}

Following the lines in [29] also the observability condition can be stated in terms of polynomials.

Proposition 2: The system (10) is observable if and only if

$$
\operatorname{deg}_{s} a(\delta, s)=n
$$

Sketch of the proof: If the system is not observable one obtains, by eliminating the state variables in (10), see Remark 1 and Appendix I, an input-output equation of the form

$$
\begin{aligned}
y^{(r)}(t)= & \varphi\left(\left\{y^{(k)}(t-i), u^{(l)}(t-j)\right.\right. \\
& 0 \leq k \leq r-1 ; 0 \leq l \leq r ; i, j \geq 0\})
\end{aligned}
$$

where $r<n$ and then $\operatorname{deg}_{s} a(\delta, s)=r$.

\section{MODEl MATCHING PROBLEM}

The transfer function approach to the model matching problem is the most natural, in comparison with the state space approaches. For the nonlinear system without delays it was recently considered in [18]. As in linear case, it is stated as the equality of the transfer functions of the model and of the compensated system. Here, we extend some basic ideas of [18] to the time-delay case.

Consider a nonlinear system $F$ and a model $G$ described by the transfer functions

$$
\begin{aligned}
F(\delta, s) & =\frac{b_{F}(\delta, s)}{a_{F}(\delta, s)} \\
G(\delta, s) & =\frac{b_{G}(\delta, s)}{a_{G}(\delta, s)}
\end{aligned}
$$

respectively. Find a (proper) feedforward compensator $R$, described by the transfer function

$$
R(\delta, s)
$$

such that the transfer function of the compensated system coincides with that of the model $G$

$$
G(\delta, s)=F(\delta, s) \cdot R(\delta, s)
$$

Proposition 3: Given $F(\delta, s) \neq 0$ and $G(\delta, s)$, there exists a feedforward compensator $R(\delta, s)$ which solves the model matching problem if $a_{R}(\delta, s) \mathrm{d} u-b_{R}(\delta, s) \mathrm{d} v$ is integrable, where $\frac{b_{R}(\delta, s)}{a_{R}(\delta, s)}=F^{-1}(\delta, s) \cdot G(\delta, s)$.

Proof: By the transfer function algebra [12] we get

$$
G(\delta, s)=F(\delta, s) \cdot R(\delta, s)
$$

Hence, the compensator

$$
R(\delta, s)=F^{-1}(\delta, s) \cdot G(\delta, s)=\frac{b_{R}(\delta, s)}{a_{R}(\delta, s)}
$$

Clearly, the existence of such a compensator is determined by the integrability of the compensator's equation $a_{R}(\delta, s) \mathrm{d} u=$ $b_{R}(\delta, s) \mathrm{d} v$.

Example 3: Given the system $F$

$$
\dot{y}(t)=\dot{u}(t-1)+u^{2}(t-2)
$$


with the transfer function

$$
F(\delta, s)=\frac{\delta s+2 u(t-2) \delta^{2}}{s}
$$

Consider the following three models

$$
\begin{aligned}
G(\delta, s) & =\frac{\delta}{s} \\
G^{\prime}(\delta, s) & =\frac{\delta}{s+1} \\
G^{\prime \prime}(\delta, s) & =\frac{\delta}{s+2 y(t)}
\end{aligned}
$$

By (7) and (3) we get the following transfer functions of the compensators

$$
\begin{aligned}
R(\delta, s) & =\frac{s}{\delta s+2 u(t-2) \delta^{2}} \cdot \frac{\delta}{s} \\
& =\frac{\delta}{\delta s+2 u(t-2) \delta^{2}} \\
R^{\prime}(\delta, s) & =\frac{s}{\delta s+2 u(t-2) \delta^{2}} \cdot \frac{\delta}{s+1} \\
& =\frac{\delta s}{(s+1)\left(\delta s+2 u(t-2) \delta^{2}\right)} \\
R^{\prime \prime}(\delta, s) & =\frac{\delta}{\delta s+2 u(t-2) \delta^{2}} \cdot \frac{\delta+2 y(t)}{\delta s-\frac{\dot{y}(t)}{y(t)} \delta} \\
& =\frac{\dot{y}(t)}{\left(s+2 y(t)-\frac{1}{y(t)}\right)\left(\delta s+2 u(t-2) \delta^{2}\right)}
\end{aligned}
$$

While $R(\delta, s)$ and $R^{\prime}(\delta, s)$ result in the integrable compensators

$$
\begin{aligned}
R: \dot{u}(t)= & -u^{2}(t-1)+v(t) \\
R^{\prime}: \quad \ddot{u}(t)= & -2 u(t-1) \dot{u}(t-1)-\dot{u}(t)- \\
&
\end{aligned}
$$

$R^{\prime \prime}(\delta, s)$ does not.

Remark that in multiplying transfer functions one always has to follow the rule (3) which, in general, yields a different result from the standard multiplication, as can be seen for instance in the case of $R^{\prime \prime}(\delta, s)$.

Remark 3: Note that in contrast to what happens in the linear case, a class of nonlinear systems for which the solution in terms of a feedforward compensator exists is, due to the integrability condition, quite restricted.

\section{A. Properness of the compensator}

Obviously, we are interested in finding a solution in a class of proper compensators. However, in comparison to what happens in the case of systems without delays [18] here, it is not sufficient to restrict just the relative degrees of the model and of the system by the standard inequality

$$
\operatorname{rel} \operatorname{deg}_{s} G(\delta, s) \geq \operatorname{reldeg}_{s} F(\delta, s)
$$

even in the linear case.

Example 4: Consider the system

$$
F(\delta, s)=\frac{\delta^{2}}{s-\delta}
$$

and the model

$$
G(\delta, s)=\frac{\delta}{s^{2}}
$$

However, even if $\operatorname{rel}_{\operatorname{deg}_{s}} G(\delta, s)=2 \geq \operatorname{rel}_{\operatorname{deg}_{s}} F(\delta, s)=1$ the compensator

$$
R(\delta, s)=F^{-1}(\delta, s) \cdot G(\delta, s)=\frac{s-\delta}{\delta^{2}} \cdot \frac{\delta}{s^{2}}=\frac{s-\delta}{\delta s^{2}}
$$

is nonproper

$$
\begin{aligned}
\ddot{u}(t-1) & =\dot{v}(t)-v(t-1) \\
\ddot{u}(t) & =\dot{v}(t+1)-v(t)
\end{aligned}
$$

Here, the necessary and sufficient condition, under which the compensator becomes proper, is more involved. Clearly, to satisfy the propernes of the compensator $R$ the highest derivative of its output has to depend only on the past values of its output, input and their derivatives

$$
\begin{aligned}
\mathrm{d} u^{(k)}(t-l) \in & \operatorname{span}_{\mathcal{K}}\left\{\mathrm{d} u^{\left(i_{1}\right)}(t-j), \mathrm{d} v^{\left(i_{2}\right)}(t-j) ;\right. \\
& \left.0 \leq i_{1} \leq k-1 ; 0 \leq i_{2} \leq k, j \geq l\right\}
\end{aligned}
$$

for some $l \geq 0$.

Remark 4: Note that in the linear time-delay systems the properness of the compensator (7) is satisfied by the requirement that $a_{n_{R}}(\delta)$, which is the leading coefficient of $a_{R}(\delta, s)$ in $s$, contains a nonzero constant term [30]. That is, $\operatorname{low}_{\operatorname{deg}_{\delta}} a_{n_{R}}(\delta)=0$. However, in that case the solution is, in general, given by a compensator from the class of systems of a neutral type. Such solutions are out of the scope of this paper, as we restricted our attention to systems, models and compensators respectively of the form (1). From this point of view, the condition (8) means that the leading coefficient contains only a nonzero constant term; that is, $\operatorname{deg}_{\delta} a_{n_{R}}(\delta)=0$.

Clearly, the condition (8), in terms of the transfer function (7), means that in addition to $\operatorname{deg}_{s} a_{R}(\delta, s) \geq \operatorname{deg}_{s} b_{R}(\delta, s)$ also powers of $\delta$ in $a_{R}(\delta, s)$ and $b_{R}(\delta, s)$ have to be taken into account. Namely

$$
\operatorname{deg}_{\delta} a_{n_{R}}(\delta) \leq \min \left\{\operatorname{low} \operatorname{deg}_{\delta} a_{R}(\delta, s), \operatorname{low}_{\operatorname{deg}_{\delta}} b_{R}(\delta, s)\right\}
$$

where $a_{n_{R}}(\delta)$ is the leading coefficient of $a_{R}(\delta, s)$ in $s$.

When coming back to the transfer functions $F(\delta, s)$ and $G(\delta, s)$ one concludes that

Proposition 4: $R(\delta, s)$ is proper (causal) if and only if

- $\operatorname{rel} \operatorname{deg}_{s} G(\delta, s) \geq \operatorname{rel} \operatorname{deg}_{s} F(\delta, s)$

- $\mu_{F} \leq \min \left\{\operatorname{low}_{\operatorname{deg}_{\delta}} b_{F}(\delta, s), \operatorname{low}_{\operatorname{deg}_{\delta}} b_{G}(\delta, s)\right\}$

where $\mu_{F}$ is the relative shift of the system $F$.

Proof: For $\operatorname{rel} \operatorname{deg}_{s} G(\delta, s) \geq \operatorname{reldeg}_{s} F(\delta, s)$ it follows the same line as in [18]. For the second part note firstly that $\mu_{F}=\operatorname{deg}_{\delta} b_{m_{F}}(\delta)$ where $b_{m_{F}}(\delta)$ is the leading coefficient of $b_{F}(\delta, s)$ in $s$. Now, from (7) it follows that

$$
\begin{aligned}
\operatorname{deg}_{\delta} a_{n_{R}}(\delta) & =\operatorname{deg}_{\delta} b_{m_{F}}(\delta)+\operatorname{deg}_{\delta} a_{n_{G}}(\delta) \\
\operatorname{low}_{\operatorname{deg}_{\delta}} a_{R}(\delta) & =\operatorname{low} \operatorname{deg}_{\delta} b_{F}(\delta)+\operatorname{low} \operatorname{deg}_{\delta} a_{G}(\delta) \\
\text { low } \operatorname{deg}_{\delta} b_{R}(\delta) & =\operatorname{low} \operatorname{deg}_{\delta} a_{F}(\delta)+\operatorname{low} \operatorname{deg}_{\delta} b_{G}(\delta)
\end{aligned}
$$

where $a_{n_{G}}(\delta)$ is the leading coefficient of $a_{G}(\delta, s)$ in $s$. Since both the system and the model are considered to 
be of the form (1) note, however, that $\operatorname{deg}_{\delta} a_{n_{G}}(\delta)=$

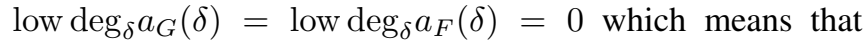
$\mu_{F} \leq \min \left\{\operatorname{low}_{\operatorname{deg}} b_{F}(\delta, s), \operatorname{low}_{\operatorname{deg}_{\delta}} b_{G}(\delta, s)\right\}$ is equivalent to (9).

Note that in Example 4 we have $\mu_{F}=2$, $\operatorname{low} \operatorname{deg}_{\delta} b_{F}(\delta, s)=2$ and $\operatorname{low}_{\operatorname{deg}_{\delta}} b_{G}(\delta, s)=1$ and in Example 3 we have $\mu_{F}=1$, $\operatorname{low}_{\operatorname{deg}_{\delta}} b_{F}(\delta, s)=1$ and for all $G, G^{\prime}$ and $G^{\prime \prime} \operatorname{low}_{\operatorname{deg}_{\delta}} b_{G}(\delta, s)=\operatorname{low}_{\operatorname{deg}_{\delta}} b_{G^{\prime}}(\delta, s)=$ low $\operatorname{deg}_{\delta} b_{G^{\prime \prime}}(\delta, s)=1$.

\section{Conclusions}

In this paper, several problems are discussed within the transfer function formalism of nonlinear time-delay systems. Mainly, the structural properties of the transfer functions and the model matching problem. In the model matching problem it was shown that the existence of the compensator requires a restrictive integrability condition and that its properness requires to restrict not only the relative degrees, as in the case without delays [18], but the relative shift and a zero structure of both the system and the model as well. A preliminary result relating the state elimination problem for the nonlinear time-delay case was also given.

In conclusion, the paper depicts basic ideas in a quite wide range of problems relating the nonlinear time-delay systems and opens the others that are worth to be studied, as for instance finding a solution to the model matching problem of nonlinear time-delay systems allowing the compensator to be a system of a neutral type, or considering the more general class of feedback compensators, etc.

\section{APPENDIX I}

\section{STATE ELIMINATION FOR SISO NONLINEAR TIME-DELAY} SYSTEMS

Consider the nonlinear time-delay system of the form

$$
\begin{aligned}
& \dot{x}(t)=f(\{x(t-i), u(t-j) ; i, j \geq 0\}) \\
& y(t)=g(\{x(t-i), u(t-j) ; i, j \geq 0\})
\end{aligned}
$$

where $x \in \mathbf{R}^{n}, u \in \mathbf{R}$ and $y \in \mathbf{R}$.

To find an input-output equation for the system (10) we will try to carry over the idea of the state elimination procedure known for the systems without delays, where an input-output representation is constructed by applying a suitable change of coordinates [6], also to the time-delay case. However, as the system (10) is the subject of two operators, in comparison to what happens in the case of systems without delays we may not be able to find such a change of coordinates.

Example 5: Consider the system

$$
\begin{aligned}
& \dot{x}(t)=u(t) \\
& y(t)=x(t) x(t-1)
\end{aligned}
$$

Following the lines of [6]

$$
\dot{y}(t)=u(t) x(t-1)+x(t) u(t-1)
$$

However, one cannot go any further, since from $y(t)=$ $x(t) x(t-1)$ one cannot express $x(t)$.

Remark 5: It was shown in [1] that it is possible to derive $N$ independent equations over $\mathcal{K}$ in $N$ variables $x_{i}(t-j)$, $i=1, \ldots, n, j \geq 0$, for some $N$ which may be greater than $n$. For instance, in the previous example one considers

$$
\begin{aligned}
y(t) & =x(t) x(t-1) \\
y(t-1) & =x(t-1) x(t-2) \\
\dot{y}(t-1) & =u(t-1) x(t-2)+x(t-1) u(t-2)
\end{aligned}
$$

However, in that case it yields, in general, an input-output equation representing a system of a neutral type, i.e. not being of the form (1). Thus, for our purpose a stronger result is needed.

In what follows we present the state elimination procedure extended to a class of systems with delays.

\section{State elimination procedure:}

Let $s$ denote the minimum nonnegative integer such that

$$
\operatorname{rank}_{\mathcal{K}[\delta]}\left(\begin{array}{c}
c_{0}(\delta) \\
\vdots \\
c_{s-1}(\delta)
\end{array}\right)=\operatorname{rank}_{\mathcal{K}[\delta]}\left(\begin{array}{c}
c_{0}(\delta) \\
\vdots \\
c_{s}(\delta)
\end{array}\right)
$$

where $c_{i}(\delta) \in \mathcal{K}^{1 \times n}[\delta]$ and $d_{i}(\delta) \in \mathcal{K}[\delta]$ are such that

$$
\mathrm{d} y^{(i)}(t)=c_{i}(\delta) \mathrm{d} x(t)+d_{i}(\delta) \mathrm{d} u(t)
$$

for $i=0, \ldots, s$. If $c_{0}(\delta)=0$ we define $s=0$ and $y(t)=$ $g(\cdot)$ is the input-output equation. Note that

$$
\left(\begin{array}{c}
c_{0}(\delta) \\
\vdots \\
c_{s-1}(\delta)
\end{array}\right)
$$

is, in fact, an observability matrix and if $s<n$ the system (10) is not observable.

Clearly, to eliminate the state variables in $y^{(s)}(t)=g^{(s)}(\cdot)$ we must have that $c(\delta) \in \operatorname{span}_{\mathcal{K}[\delta]}\left\{c_{0}(\delta), \ldots, c_{s-1}(\delta)\right\}$. Then the input-output relation we are looking for can be found. That is

$$
y^{(s)}(t)=\varphi\left(\left\{y^{(k)}(t-i), u^{(l)}(t-j)\right\}\right)
$$

where $0 \leq k \leq s-1 ; 0 \leq l \leq s ; i, j \geq 0$.

Example 6: Consider the system

$$
\begin{aligned}
\dot{x}_{1}(t) & =x_{1}(t) x_{2}(t-1) \\
\dot{x}_{2}(t) & =x_{2}(t) u(t) \\
y(t) & =1 / x_{1}(t)
\end{aligned}
$$

We have $\dot{y}(t)=-x_{2}(t-1) / x_{1}(t), \ddot{y}(t)=-x_{2}(t-1) u(t-$ $1) / x_{1}(t)+x_{2}^{2}(t-1) / x_{1}(t)$ and

$$
\begin{aligned}
& x_{1}(t)=1 / y(t) \\
& x_{2}(t)=-\dot{y}(t+1) / y(t+1)
\end{aligned}
$$

Finally

$$
\ddot{y}(t)=\dot{y}(t) u(t-1)-\dot{y}^{2}(t) / y(t)
$$




\section{REFERENCES}

[1] M. Anguelova and B. Wennberg, State elimination and identifiability of the delay parameter for nonlinear time-delay systems, Automatica, vol. 44, 2008, pp 1373-1378.

[2] E. Aranda-Bricaire, Ü. Kotta and C. Moog, Linearization of discretetime systems, SIAM Journal of Control Optimization, vol. 34, 1996, pp 1999-2023.

[3] M. Bronstein and M. Petkovšek, An introduction to pseudo-linear algebra, Theoretical Computer Science, vol. 157, 1996, pp 3-33.

[4] F. Chyzak, A. Quadrat and D. Robertz, Effective algorithms for parametrizing linear control systems over Ore algebras, Applicable Algebra in Engineering, Communications and Computing, vol. 16, 2005, pp 319-376.

[5] F. Chyzak and B. Salvy, Non-commutative elimination in Ore algebras proves multivariate identities, Journal of Symbolic Computation, vol. 26, 1998, pp 187-227.

[6] G. Conte, C.H. Moog and A.M. Perdon, Algebraic Methods for Nonlinear Control Systems. Theory and Applications, Springer-Verlag, London, 2nd edition, 2007.

[7] M. Fliess, Une interprétation algébrique de la transformation de Laplace et des matrices de transfert, Linear Algebra and its Applications, vol. 203, 1994, pp 429-442.

[8] M. Fliess, J. Lévine, P. Martin and P. Rouchon, Flatness and defect of non-linear systems: introductory theory and examples, Int. J. Control vol. 61, 1995, pp 1327-1361.

[9] M. Fliess and H. Mounier, Controllability and observability of linear delay systems: an algebraic approach, ESAIM: Control, Optimisation and Calculus of Variations, vol. 3, 1998, pp 301-314.

[10] M. Halás, An algebraic framework generalizing the concept of transfer functions to nonlinear systems, Automatica, vol. 44, 2008, pp 11811190.

[11] Halás, M.: Nonlinear time-delay systems: a polynomial approach using Ore algebras. In: Loiseau, J.J., Michiels, W., Niculescu, S., Sipahi, R. (eds.) Topics in Time-Delay Systems: Analysis, Algorithms and Control. Lecture Notes in Control and Information Sciences. Springer, to appear (2009)

[12] M. Halás, "Ore algebras: a polynomial approach to nonlinear timedelay systems", 9th IFAC Workshop Time-Delay Systems, Nantes, France, 2007.

[13] M. Halás and M. Huba, "Symbolic computation for nonlinear systems using quotients over skew polynomial ring", 14th Mediterranean Conference on Control and Automation, Ancona, Italy, 2006.

[14] M. Halás and Ü. Kotta, "Extension of the concept of transfer function to discrete-time nonlinear control systems", European Control Conference, Kos, Greece, 2007.

[15] M. Halás and Ü. Kotta, Transfer Functions of Discrete-time Nonlinear Control Systems, Proc. Estonian Acad. Sci. Phys. Math., vol. 56, 2007, pp 322-335.

[16] M. Halás and Ü. Kotta, "Synthesis of Observers for Nonlinear Systems: A Polynomial Approach", 47th Conference on Decision and Control, Cancun, Mexico, 2008.

[17] M. Halás and Ü. Kotta, "Realization problem of SISO nonlinear systems: a transfer function approach", 7th IEEE International Conference on Control \& Automation, Christchurch, New Zealand, submitted (2009).

[18] M. Halás, Ü. Kotta and C.H. Moog, ’Transfer function approach to the model matching problem of nonlinear systems", 17th IFAC World Congress, Seoul, Korea, 2008.

[19] J. Johnson, Kähler differentials and differential algebra, Annals of Mathematics, vol. 89, 1969, pp 92-98.

[20] Ü. Kotta, ’Irreducibility conditions for nonlinear input-output difference equations", 39th IEEE Conf. on Decision and Control, Sydney, Australia, 2000.

[21] Z. Li, M. Ondera and H. Wang, "Simplifying skew fractions modulo differential and difference relations", International Symposium on Symbolic and Algebraic Computation ISSAC, Linz, Austria, 2008.

[22] L.A. Márquez-Martínez, Note sur l'accessibilité des systèmes non linéaires à retards, Comptes Rendus de l'Academie des Sciences Paris, vol. 329, 1999, pp 545-550.

[23] L.A. Márquez-Martínez and C.H. Moog, "Accessibility of nonlinear time-delay systems", 40th IEEE Conf. on Decision and Control, Orlando, Florida USA, 2001.

[24] L.A. Márquez-Martínez and C.H. Moog, Input-Output Feedback Linearization of Time-Delay Systems, IEEE Transactions on Automatic Control, vol. 49, 2004, pp 781-786.
[25] L.A. Márquez-Martínez, C.H. Moog and M. Velasco-Villa, The structure of nonlinear time-delay systems, Kybernetika, vol. 36, 2000, pp 53-62.

[26] C.H. Moog and R. Castro-Linares, M. Velasco-Villa and L.A. Márquez-Martínez, The disturbance decoupling problem for timedelay nonlinear systems, IEEE Transactions on Automatic Control, vol. 45, 2000, pp 305-309.

[27] O. Ore, Linear equations in non-commutative fields, Annals of Mathematics vol. 32, 1931, pp 463-477.

[28] O. Ore, Theory of non-commutative polynomials, Annals of Mathematics vol. 34, 1933, pp 480-508.

[29] A.M. Perdon, C.H. Moog and G. Conte, "The pole-zero structure of nonlinear control systems", 7th IFAC Symposium NOLCOS, Pretoria, South Africa, 2007.

[30] P. Picard, J.F. Lafay and V. Kučera, Model Matching for Linear Systems with Delays and 2D Systems, Automatica, vol. 34, 1998 pp 183-191.

[31] X. Xia and L.A. Márquez-Martínez, P. Zagalak and C.H. Moog, Analysis of nonlinear time-delay systems using modules over noncommutative rings, Automatica, vol. 38, 2002, pp 1549-1555.

[32] Y. Zheng and L. Cao, Transfer function description for nonlinear systems, Journal of East China Normal University (Natural Science), vol. 2, 1995, pp 15-26.

[33] Y. Zheng, J. Willems and C. Zhang, A polynomial approach to nonlinear system controllability, IEEE Transactions on Automatic Control, vol. 46, 2001, pp 1782-1788.

[34] Y. Zheng, J. Willems and C. Zhang, "Common factors and controllability of nonlinear systems", 36th IEEE Conf. on Decision and Control, San Diego, California USA, 1997. 